\title{
Topological properties of full groups
}

\author{
JOHN KITTRELL $† \S$ and TODOR TSANKOV $\$$ I \\ $\dagger$ Mathematical Sciences Building 6363, University of California, Los Angeles, \\ CA 90095, USA \\ (e-mail: jw.kittrell@gmail.com) \\ \$ Department of Mathematics 253-37, California Institute of Technology, Pasadena, \\ CA 91125, USA \\ (e-mail: todor@math.jussieu.fr)
}

(Received 4 September 2007 and accepted in revised form 9 January 2009)

\begin{abstract}
We study full groups of countable, measure-preserving equivalence relations. Our main results include that they are all homeomorphic to the separable Hilbert space and that every homomorphism from an ergodic full group to a separable group is continuous. We also find bounds for the minimal number of topological generators (elements generating a dense subgroup) of full groups allowing us to distinguish between full groups of equivalence relations generated by free, ergodic actions of the free groups $\mathbf{F}_{m}$ and $\mathbf{F}_{n}$ if $m$ and $n$ are sufficiently far apart. We also show that an ergodic equivalence relation is generated by an action of a finitely generated group if an only if its full group is topologically finitely generated.
\end{abstract}

\section{Introduction}

The study of measure-preserving actions of countable groups on standard probability spaces up to orbit equivalence was initiated by Dye in the 1950s and since then the subject has become an important meeting point of ergodic theory, operator algebras, and Borel equivalence relations. This paper concentrates on the study of one invariant of orbit equivalence, namely, the full group of the orbit equivalence relation.

Let $X$ be a standard Borel space and $\mu$ a non-atomic, Borel, probability measure on it. Denote by $\operatorname{Aut}(X, \mu)$ the group of all measure-preserving automorphisms of $(X, \mu)$ (modulo null sets). An equivalence relation $E$ on $X$ is called countable if all of its equivalence classes are countable, finite if all of its equivalence classes are finite, and

$\S$ Current address: Knightsbridge Asset Management, LLC, 660 Newport Center Drive, Suite 460, Newport Beach, CA 92660, USA.

II Current address: Equipe d'Analyse, boîte 186, Université Pierre et Marie Curie, 4, Place Jussieu, 75252 Paris Cedex 05, France. 
aperiodic if all of its equivalence classes are infinite. Say that $E$ is measure-preserving if every Borel automorphism $T$ of $X$ which preserves $E$ is measure-preserving, i.e.

$$
(\forall x \in X \quad T x E x) \Longrightarrow T \text { preserves } \mu .
$$

All equivalence relations below are assumed countable, Borel, and measure-preserving. The full group of $E$, denoted by $[E]$, is defined by

$$
[E]=\{T \in \operatorname{Aut}(X, \mu): T x E x \text { for almost every } x \in X\} .
$$

By a classical result of Feldman and Moore [6], every measure-preserving equivalence relation $E$ is the orbit equivalence relation of some measure-preserving group action $\Gamma \curvearrowright X$, where $\Gamma$ is a countable group. If $\Phi \subseteq \operatorname{Aut}(X, \mu)$ is a countable set of automorphisms, write $E_{\Phi}^{X}$ for the equivalence relation generated by $\Phi$ and $[\Phi]$ for the full group $\left[E_{\Phi}^{X}\right]$. If $\Phi=\{T\}$ is a singleton, write $E_{T}^{X}$ instead of $E_{\{T\}}^{X}$ and [T] instead of $[\{T\}]$.

An equivalence relation $E$ is called ergodic if every measurable $E$-invariant set is either null or co-null. Two equivalence relations $E$ and $F$ (on measure spaces $(X, \mu)$ and $(Y, v)$, respectively) are isomorphic if there exists a measure-preserving isomorphism $f: X \rightarrow Y$ such that

$$
x_{1} E x_{2} \Longleftrightarrow f\left(x_{1}\right) F f\left(x_{2}\right) \text { for almost every } x_{1}, x_{2} \in X .
$$

Two measure-preserving countable group actions $\Gamma \curvearrowright X$ and $\Delta \curvearrowright Y$ are orbit equivalent if their orbit equivalence relations are isomorphic. An equivalence relation is called hyperfinite if it can be written as an increasing union of finite equivalence relations or, equivalently, is generated by a single automorphism. Dye proved that all ergodic, hyperfinite equivalence relations are isomorphic and Ornstein and Weiss showed that all equivalence relations generated by amenable groups are hyperfinite; in particular, all ergodic actions of amenable groups are orbit equivalent (see [16] for proofs of these facts). For non-amenable groups, however, there are many examples of non-orbit equivalent actions (both actions of different groups and different actions of the same group). Finding invariants for orbit equivalence has proved to be difficult and there are many results which indicate that satisfactory complete invariants in fact do not exist. (It follows from the work of Gaboriau and Popa [8], Törnquist [29], and Kechris [15] that actions of the free group $\mathbf{F}_{2}$, up to orbit equivalence, are not classifiable by countable structures. Recently this was extended to groups containing $\mathbf{F}_{2}$ by Ioana [13] and Kechris [15].)

The presence of a measure allows one to define a topology on the full groups which greatly facilitates their study. There are two group topologies on $\operatorname{Aut}(X, \mu)$, introduced by Halmos, which are relevant for us. Recall that the measure algebra of $(X, \mu)$, denoted by $\mathrm{MALG}_{\mu}$, is the collection of all measurable subsets of $X$ modulo null sets. It becomes an abelian group under the operation of symmetric difference and the metric defined by

$$
\rho(A, B)=\mu(A \triangle B)
$$

is invariant under the group operation. The group $\operatorname{Aut}(X, \mu)$ acts faithfully by topological group automorphisms on $\mathrm{MALG}_{\mu}$. Both topologies on $\operatorname{Aut}(X, \mu)$ that we consider are given by this action: the weak topology is the topology of pointwise convergence on $\mathrm{MALG}_{\mu}$ and the uniform topology is the topology of uniform convergence. Both 
topologies are completely metrizable; the first is separable and the second is not. A metric compatible with the uniform topology, which is moreover invariant under group multiplication from both sides, is given by

$$
d(T, S)=\mu(\{T \neq S\}) .
$$

The full groups are closed subgroups in $\operatorname{Aut}(X, \mu)$ in the uniform topology and they turn out to be separable, hence Polish. Unless otherwise mentioned, we assume them to be equipped with this Polish topology. For all of this, see [15].

Full groups were first considered by Dye and our main motivation to study them comes from the following theorem (see [15] for a recent exposition of the proof).

THEOREM 1.1. (Dye [4]) Let $E$ and $F$ be two countable, measure-preserving, ergodic equivalence relations on the standard probability space $(X, \mu)$. Then the following are equivalent:

(i) $E$ and $F$ are isomorphic;

(ii) $[E]$ and $[F]$ are isomorphic (algebraically);

(ii) there exists $f \in \operatorname{Aut}(X, \mu)$ such that $f[E] f^{-1}=[F]$.

Moreover, every algebraic isomorphism between $[E]$ and $[F]$ is realized by a conjugacy.

Theorem 1.1 suggests that the algebraic structure of full groups is rich enough to 'remember' the topology since, by the last assertion, every algebraic automorphism of an ergodic full group is automatically a homeomorphism. We pursue this point further in $\S 3$ where we prove one of our main results (cf. Theorem 3.1).

THEOREM 1.2. Let E be an ergodic, measure-preserving, countable equivalence relation. Then every homomorphism $f:[E] \rightarrow G$, where $G$ is a separable topological group, is automatically continuous. In particular, the uniform topology is the finest separable group topology on $[E]$ and, hence, the unique Polish topology.

Hence, the structure of $[E]$ as an abstract group alone is sufficient to recover the topology and any statement about $[E]$ as a topological group can, at least in principle, be translated into a statement referring only to its algebraic structure. Automatic continuity of this type is a phenomenon which appeared recently in the work of Kechris and Rosendal [17], Rosendal and Solecki [27], and Rosendal [26] (see $\$ 3$ for more examples and further discussion). Automatic continuity also has implications for group actions on various spaces; for example, any action of a group with this property by homeomorphisms on a compact metrizable space or by linear isometries on a separable Banach space is automatically continuous.

Dye's theorem shows that full groups are complete invariants for orbit equivalence; thus, in order to prove that two equivalence relations are non-isomorphic, it suffices to find a (topological group) property of their full groups which differentiates them. The only known (to the authors) result of that flavor to date is the following.

TheOREM 1.3. (Giordano and Pestov [9]) Let E be a countable, measure-preserving, ergodic equivalence relation. Then $[E]$ is hyperfinite if and only if $[E]$ is extremely amenable. 
Recall that a topological group is called extremely amenable if every time it acts continuously on a compact space, the action has a fixed point. The property of extreme amenability has recently received a lot of attention; see Pestov [25] for a discussion and references.

Combining Theorems 1.2 and 1.3 yields the following result.

COROLlaRY 1.4. Let $E$ be ergodic and hyperfinite. Then any action of $[E]$ by homeomorphisms on a compact, metrizable space has a fixed point.

One should contrast this with the fact that any discrete group admits a free action on a compact space (see [25]).

Going back to orbit equivalence, one could perhaps hope to distinguish the full groups as topological spaces alone (forgetting the group structure). This, however, turns out to be impossible as they are all homeomorphic (cf. Corollary 2.5).

THEOREM 1.5. Let E be a countable, measure-preserving equivalence relation on the standard probability space $(X, \mu)$ which is not equality almost everywhere. Then the full group $[E]$ with the uniform topology is homeomorphic to the Hilbert space $\ell^{2}$.

It was previously known that full groups are contractible. This follows from the argument of Keane [14] and was first explicitly noted by Danilenko [2].

Another possible invariant (suggested by Kechris [15]) one could look at is the number of topological generators of $[E]$, denoted by $t([E])$ (i.e. the minimal number of generators of a dense subgroup of $[E])$. Since the group $[E]$ is separable, we always have $t([E]) \leq \aleph_{0}$. It is also easily seen that $t([E]) \geq 2$ if $E$ is not trivial. Indeed, if $E$ has a positive set of classes of size greater than two, then $[E]$ is non-abelian and if $E$ is generated by a non-trivial involution, $[E]$ is isomorphic to $\left(\mathrm{MALG}_{\mu}, \triangle\right)$ which is also not monothetic. The next observation is that if $\Gamma \leq[E]$ is countable and dense, then $\Gamma$ must generate $E$ (since $[\Gamma]$ is closed and by density, $[\Gamma]=[E]$ ). Now Gaboriau's theory of cost [7] (see also Kechris and Miller [16]) allows one to conclude that if $E$ is generated by a free action of $\mathbf{F}_{n}$, then $t([E]) \geq n$. (In this case, it is not hard to show the slightly better bound $t([E]) \geq n+1$ (Miller); cf. Corollary 4.12.) So, to show that $t([E])$ is a non-trivial invariant, it suffices to find equivalence relations $E$ for which $t([E])$ is finite. Our first result in this direction is an upper bound for $t([E])$ for ergodic, hyperfinite $E$ (cf. Theorem 4.2).

THEOREM 1.6. Let E be ergodic and hyperfinite. Then $t([E]) \leq 3$.

Using this and an inductive procedure, we further show the following (cf. Corollary 4.11).

THEOREM 1.7. Let $E$ be an ergodic equivalence relation on $(X, \mu)$. Then the following are equivalent:

(i) E can be generated by an action of a finitely generated group;

(ii) E has finite cost;

(iii) $[E]$ is topologically finitely generated. 
Specific calculations for $t([E])$, for example, for $E$ generated by free, ergodic actions of free groups, would allow us to distinguish those equivalence relations. It is known that free actions of free groups with different numbers of generators are orbit inequivalent but the only known way to show that is by using cost (see Gaboriau [7]). Here we provide estimates for $t([E])$ which distinguish free, ergodic actions of $\mathbf{F}_{m}$ and $\mathbf{F}_{n}$ when $m$ and $n$ are sufficiently far apart. The proof of the lower bound, however, still depends on Gaboriau's results on cost (cf. Corollary 4.12).

THEOREM 1.8. Let the equivalence relation $E$ be generated by a free, ergodic action of the free group $\mathbf{F}_{n}, n \geq 1$. Then

$$
n+1 \leq t([E]) \leq 3 n+3 .
$$

It would be interesting to sharpen those estimates as well as find a proof for the lower bound independent of cost.

The organization of this paper is as follows. In $\S 2$, we prove Theorem 1.5; in $\S 3$, we prove the automatic continuity results; and, finally, in $\$ 4$, we discuss topological generators.

In what follows, $(X, \mu)$ is a standard probability space and all equivalence relations which we consider are countable and measure-preserving. We habitually ignore null sets if there is no danger of confusion. If $A \subseteq X, A^{\complement}$ denotes the complement of $A$. If $T \in \operatorname{Aut}(X, \mu), \operatorname{supp} T$ denotes the support of $T$, i.e. the set $\{x \in X: T x \neq x\}$.

\section{Full groups are homeomorphic to $\ell^{2}$}

Identifying the topological type of big symmetry groups has been an ongoing enterprise for the last few decades. During that time, infinite-dimensional topology has developed many tools which allow us to do that. Recall that a topological space $Y$ is called an absolute (neighborhood) retract (abbreviated $\mathrm{A}(\mathrm{N}) \mathrm{R}$ ) if every time it embeds as a closed subspace of a normal space $Z$, the image of the embedding is a (neighborhood) retract of $Z$. The prototypical examples of absolute retracts (ARs) are the convex subsets of normed linear spaces. A basic fact is that an ANR is an AR if and only if it is contractible [30, Theorem 5.2.15]. We use the book by van Mill [30] as a basic reference for infinitedimensional topology.

A basic tool that allows to show that a given Polish group is homeomorphic to $\ell^{2}$ is a theorem of Dobrowolski and Toruńczyk [3] which states that every non-locally compact Polish group whose underlying topological space is an ANR is homeomorphic to an $\ell^{2}$ manifold. Moreover, every contractible $\ell^{2}$-manifold is in fact homeomorphic to $\ell^{2}[\mathbf{1}$, Ch. IX, Theorem 7.3]. Currently many big Polish groups are known to be homeomorphic to $\ell^{2}$. Examples include $\operatorname{Aut}(X, \mu)$ with the weak topology (Nhu [24]), the group of orientation-preserving homeomorphisms of the unit interval (Anderson), the isometry group of the Urysohn space (Melleray [22]), and many others.

Regarding full groups, we have the following result.

THEOREM 2.1. Let $E$ be a countable, measure-preserving equivalence relation on $(X, \mu)$. Then $[E]$ is an absolute retract. 
Proof. Keane [14] showed that $\operatorname{Aut}(X, \mu)$ is contractible in both the weak and the uniform topologies using induced transformations and the same argument shows that $[E]$ is contractible. (We discuss induced transformations below and outline his method.) In order to verify that $[E]$ is an ANR, it suffices to produce a basis $\mathcal{B}$ for its topology with the following property:

every non-empty finite intersection of elements of $\mathcal{B}$ is homotopically trivial

(see [30, Theorem 5.2.12]). We prove that the basis consisting of the open balls of the uniform metric $d$ defined by (1.1) has this property.

Let

$$
\operatorname{PER}=\left\{T \in \operatorname{Aut}(X, \mu): \exists n T^{n}=1\right\} .
$$

The Rokhlin lemma implies that PER $\cap[E]$ is uniformly dense in $[E]$. If $\xi$ is a finite partition of $X$, denote by $\hat{\xi}$ the finite Boolean algebra generated by $\xi$. We say that a set $B \in \mathrm{MALG}_{\mu}$ is independent of $\xi$ if

$$
\text { for all } A \in \xi \quad \mu(A \cap B)=\mu(A) \mu(B) .
$$

Denote by $I$ the closed unit interval $[0,1]$ and by $S^{n}$ the $n$-dimensional sphere. We split the proof into a sequence of lemmas.

LEMMA 2.2. For all $T \in \mathrm{PER}$ and finite partitions $\xi$ of $X$, there exists a continuous map $B: I \rightarrow$ MALG $_{\mu}$ satisfying the conditions:

(i) $\mu(B(\lambda))=\lambda$;

(ii) $\lambda \leq \lambda^{\prime} \Longrightarrow B(\lambda) \subseteq B\left(\lambda^{\prime}\right)$;

(iii) for all $\lambda \in I$, the set $B(\lambda)$ is $T$-invariant;

(iv) for all $\lambda \in I, B(\lambda)$ is independent of $\xi$.

Proof. Let $T^{n}=1$. By splitting $X$ into pieces (and refining $\xi$ appropriately), we can assume that all $x \in X$ have $T$-orbits of length exactly $n$. Let

$$
\begin{aligned}
\xi^{\prime} & =\xi \vee T \xi \vee \cdots \vee T^{n-1} \xi \\
& =\left\{A_{0} \cap T\left(A_{1}\right) \cap \cdots \cap T^{n-1}\left(A_{n-1}\right): A_{0}, A_{1}, \ldots, A_{n-1} \in \xi\right\} .
\end{aligned}
$$

The partition $\xi^{\prime}$ is clearly $T$-invariant, so we have an action of $T$ on it. Let $\left\{C_{1}, C_{2}, \ldots, C_{k}\right\} \subseteq \xi^{\prime}$ be a transversal for the $T$-orbits in $\xi^{\prime}$ and $Y=C_{1} \cup C_{2} \cup \cdots \cup C_{k}$. By refining $\xi$ if necessary, we can assume that all orbits have length $n$. There exists a map $h: I \rightarrow \operatorname{MALG}_{\mu}(Y)$ such that:

- $\mu(h(\lambda))=\lambda \cdot \mu(Y)$;

- $\lambda \leq \lambda^{\prime} \Longrightarrow h(\lambda) \subseteq h\left(\lambda^{\prime}\right)$

- $\mu\left(h(\lambda) \cap C_{i}\right)=\mu(h(\lambda)) \mu\left(C_{i}\right), i=1, \ldots, k$.

Now let

$$
B(\lambda)=\bigcup_{j=0}^{n-1} T^{j}(h(\lambda)) .
$$

It is clear that this $B$ satisfies the requirements. Continuity follows from (i) and (ii).

\section{CAMBRIDGE JDURNALS}


Let $\rho$ be any compatible metric on $S^{n}$.

LEMMA 2.3. For all $\delta>0$, there exists $m \in \mathbf{N}$, points $z_{1}, \ldots, z_{m} \in S^{n}$, and a continuous map $g: S^{n} \rightarrow \mathbf{R}^{m}$ such that for all $z \in S^{n}$, the following conditions are satisfied:

(i) for all $i, g_{i}(z) \geq 0$ and $\max _{i \leq m} g_{i}(z)=1$;

(ii) at most $n+1$ of the numbers $g_{1}(z), g_{2}(z), \ldots, g_{m}(z)$ are non-zero;

(iii) for all $i \leq m, g_{i}(z)>0 \Longrightarrow \rho\left(z, z_{i}\right)<\delta$,

where $g_{i}$ denotes the ith coordinate of $g$.

Proof. Consider $S^{n}$ as a simplicial complex such that all of its simplices have $\rho$-diameter smaller than $\delta$. Let $\left\{z_{1}, \ldots, z_{m}\right\}$ be the 0 -skeleton of the complex. Then for each $z \in S^{n}$ there is a minimal set $\beta(z) \subseteq\{1, \ldots, m\}$ with $|\beta(z)| \leq n+1$ such that $z$ belongs to the simplex $\left\{z_{i}: i \in \beta(z)\right\}$. Each $z$ can be written uniquely as a convex combination $\sum_{i \in \beta(z)} a_{i} z_{i}$. Set

$$
g_{i}(z)= \begin{cases}\frac{a_{i}}{\max _{i} a_{i}} & \text { if } i \in \beta(z), \\ 0 & \text { if } i \notin \beta(z) .\end{cases}
$$

It is easy to verify that $g$ satisfies the requirements.

Since the basis of open balls of the uniform metric $d$ is translation invariant, the following lemma suffices to verify $(*)$.

LeMmA 2.4. Let $\mathrm{B}_{r_{1}}\left(Q_{1}\right), \ldots, \mathrm{B}_{r_{k}}\left(Q_{k}\right)$ be open balls in $[E]\left(\mathrm{B}_{r_{j}}\left(Q_{j}\right)\right.$ denotes the ball with center $Q_{j}$ and radius $\left.r_{j}\right)$. Let $U=\bigcap_{j=1}^{k} \mathrm{~B}_{r_{j}}\left(Q_{j}\right)$ and suppose that $1 \in U$. Then any continuous map $f:\left(S^{n}, s_{0}\right) \rightarrow(U, 1)$, where $s_{0} \in S^{n}$, is nullhomotopic in $U$.

Proof. We will build a continuous map $F: S^{n} \times I \rightarrow U$ satisfying $F(z, 0)=f(z)$, $F(z, 1)=1$, and $F\left(s_{0}, \lambda\right)=1$ for all $z \in S^{n}, \lambda \in I$.

By the compactness of $S^{n}$, there exists $\epsilon>0$ such that

$$
f\left(S^{n}\right) \subseteq \bigcap_{i} \mathrm{~B}_{r_{i}-6 \epsilon}\left(Q_{i}\right) .
$$

Let $\delta<\epsilon /(n+1)$ be such that $1 / \delta$ is an integer. Since $f$ is uniformly continuous, there exists $\delta^{\prime}>0$ such that

$$
\text { for all } z, w \in S^{n} \quad \rho(z, w)<\delta^{\prime} \Longrightarrow d(f(z), f(w))<\delta .
$$

Apply Lemma 2.3 with $\delta=\delta^{\prime}$ to obtain points $z_{1}, \ldots, z_{m} \in S^{n}$ and a map $g: S^{n} \rightarrow \mathbf{R}^{m}$ with the properties described in the lemma. Note that by properties (i) and (iii),

$$
\text { for all } z \in S^{n} \text { there exists } i \leq m \text { such that } d\left(f(z), f\left(z_{i}\right)\right)<\delta<\epsilon \text {. }
$$

Let $T_{1}, T_{2}, \ldots, T_{m} \in \operatorname{PER} \cap[E]$ be such that $d\left(f\left(z_{i}\right), T_{i}\right)<\delta$ for all $i$. Let $\xi_{0}$ be the partition of $X$ generated by the collection

$$
\left\{\left\{f\left(z_{i}\right)=Q_{j}\right\},\left\{Q_{j}=1\right\}: i=1, \ldots, m ; j=1, \ldots, k\right\} .
$$

Apply Lemma 2.2 to $T_{1}$ and $\xi_{0}$ to obtain a map $B_{1}: I \rightarrow$ MALG $_{\mu}$. Inductively, assuming that $B_{i}$ and $\xi_{i-1}$ have been built, let $\xi_{i}$ be generated by $\xi_{i-1}$ and the collection

$$
\left\{B_{i}(\delta), B_{i}(2 \delta), \ldots, B_{i}(1)\right\}
$$


and apply Lemma 2.2 to $T_{i+1}$ and $\xi_{i}$ to obtain $B_{i+1}$. Our construction and Lemma 2.2(iv) ensures that $B_{i}(\lambda)$ is independent of $B_{j}(q \delta)$ for all integers $q \leq 1 / \delta$ and $j<i$ and all of them are independent of $\xi_{0}$. Hence, for any $A \in \hat{\xi}_{0}$ and any tuple $\left(q_{1}, q_{2}, \ldots, q_{m}\right)$ of integers with $q_{i} \leq 1 / \delta$,

$$
\begin{aligned}
\mu\left(A \cap \bigcap_{i=1}^{m} B_{i}\left(q_{i} \delta\right)\right) & =\mu\left(A \cap \bigcap_{i=1}^{m-1} B_{i}\left(q_{i} \delta\right)\right) \mu\left(B_{m}\left(q_{m} \delta\right)\right) \\
& =\cdots \\
& =\mu(A) \prod_{i} \mu\left(B_{i}\left(q_{i} \delta\right)\right) .
\end{aligned}
$$

Now define a map $h: S^{n} \times I \rightarrow \mathrm{MALG}_{\mu}$ by

$$
h(z, \lambda)=\bigcap_{i=1}^{m} B_{i}\left(1-\lambda g_{i}(z)\right) \text {. }
$$

The idea is that $h(z, \lambda)$ is 'almost invariant' under the action of $f(z)$ and 'almost independent' of the partition $\xi_{0}$. This will allow us to show that the induced transformation $f(z)_{h(z, \lambda)}$ is in $U$ and $F(z, \lambda)=f(z)_{h(z, \lambda)}$ will furnish the desired homotopy. The following claim summarizes the properties of $h$ we need.

CLAIM. The following statements hold for $h$ :

(i) $h$ is continuous;

(ii) $h(z, 0)=X, h(z, 1)=\emptyset$;

(iii) $\mu(f(z)(h(z, \lambda)) \triangle h(z, \lambda))<2 \epsilon$;

(iv) for all $A \in \hat{\xi}_{0},|\mu(h(z, \lambda) \cap A)-\mu(h(z, \lambda)) \mu(A)|<2 \epsilon$.

Proof. Statements (i) and (ii) are clear from the definition and the properties of $g$. We proceed to verify (iii) and (iv). Fix $z$ and $\lambda$ and set $T=f(z)$. Let $\alpha=\left\{i: g_{i}(z)>0\right\}$. By Lemma 2.3(ii), $|\alpha| \leq n+1$. Set $D_{i}=B_{i}\left(1-\lambda g_{i}(z)\right)$ and let

$$
C=h(z, \lambda)=\bigcap_{i \in \alpha} D_{i}
$$

By (2.1) and Lemma 2.3(iii),

$$
\text { for all } i \in \alpha \quad d\left(T, T_{i}\right)<2 \delta,
$$

so, in particular, $\mu\left(T(A) \triangle T_{i}(A)\right)<2 \delta$ for any $A \in \mathrm{MALG}_{\mu}, \quad i \in \alpha$. Using Lemma 2.2(iii), we have

$$
\begin{aligned}
\mu(T(C) \triangle C) & =\mu\left(T\left(\bigcap_{i \in \alpha} D_{i}\right) \Delta \bigcap_{i \in \alpha} D_{i}\right) \\
& \leq \sum_{i \in \alpha} \mu\left(T\left(D_{i}\right) \Delta D_{i}\right) \\
& \leq \sum_{i \in \alpha} \mu\left(T\left(D_{i}\right) \triangle T_{i}\left(D_{i}\right)\right)+\sum_{i \in \alpha} \mu\left(T_{i}\left(D_{i}\right) \triangle D_{i}\right) \\
& \leq 2|\alpha| \delta+0<2 \epsilon
\end{aligned}
$$

which verifies (iii).

\section{CAMBRIDGE JDURNALS}


Now fix $A \in \hat{\xi}_{0}$. Let for each $i \in \alpha, q_{i} \leq 1 / \delta$ be such that

$$
\left|\mu\left(D_{i} \triangle B_{i}\left(q_{i} \delta\right)\right)\right| \leq \delta .
$$

Then

$$
\begin{aligned}
\mu\left(C \triangle \bigcap_{i \in \alpha} B_{i}\left(q_{i} \delta\right)\right) & =\mu\left(\bigcap_{i \in \alpha} D_{i} \triangle \bigcap_{i \in \alpha} B_{i}\left(q_{i} \delta\right)\right) \\
& \leq \mu\left(\bigcup_{i \in \alpha} D_{i} \triangle B_{i}\left(q_{i} \delta\right)\right) \\
& \leq|\alpha| \delta<\epsilon .
\end{aligned}
$$

By (2.2),

$$
\mu\left(\bigcap_{i \in \alpha} B_{i}\left(q_{i} \delta\right)\right)=\prod_{i \in \alpha} \mu\left(B_{i}\left(q_{i} \delta\right)\right)
$$

and

$$
\mu\left(A \cap \bigcap_{i \in \alpha} B_{i}\left(q_{i} \delta\right)\right)=\mu(A) \prod_{i \in \alpha} \mu\left(B_{i}\left(q_{i} \delta\right)\right),
$$

which, together with (2.3), allow us to calculate

$$
\begin{aligned}
|\mu(A \cap C)-\mu(A) \mu(C)| & <\left|\mu\left(A \cap \bigcap_{i \in \alpha} B_{i}\left(q_{i} \delta\right)\right)-\mu(A) \mu\left(\bigcap_{i \in \alpha} B_{i}\left(q_{i} \delta\right)\right)\right|+2 \epsilon \\
& =2 \epsilon,
\end{aligned}
$$

verifying (iv).

For $T \in[E]$ and $A \in \mathrm{MALG}_{\mu}$, let $T_{A} \in[E]$ denote the induced transformation, i.e.

$$
T_{A}(x)= \begin{cases}x & \text { if } x \notin A, \\ T^{s(x)}(x) & \text { if } x \in A,\end{cases}
$$

where $s(x)$ is the least $s>0$ for which $T^{s}(x) \in A . T_{A}$ is well defined (almost everywhere) by Poincaré's recurrence lemma and as follows from Keane [14], the map $(A, T) \mapsto T_{A}$ is continuous $\mathrm{MALG}_{\mu} \times[E] \rightarrow[E]$. (From here it is not hard to see that $[E]$ is contractible. Indeed, identifying $(X, \mu)$ with $\left(I\right.$, Lebesgue measure) and defining $C: I \rightarrow$ MALG $_{\mu}$ by $C(\lambda)=[0, \lambda]$, it is immediate that the map $I \times[E] \rightarrow[E]$ given by $(\lambda, T) \mapsto T_{C(\lambda)}$ is a contraction.)

Now set

$$
F(z, \lambda)=f(z)_{h(z, \lambda)} .
$$

We only need to verify that $F\left(S^{n}, I\right) \subseteq U$. Fix $z \in S^{n}, \lambda \in I$, and $j \in\{1, \ldots, k\}$. Set $T=f(z), C=h(z, \lambda)$. Find $z_{i}$ such that $d\left(T, f\left(z_{i}\right)\right)<\epsilon$. Note also that by (iii) of the claim,

$$
\mu\left(C \cap\left\{T \neq T_{C}\right\}\right)=\mu\left(C \backslash T^{-1}(C)\right)=\mu(T(C) \backslash C)<2 \epsilon .
$$


Using (iv) of the claim, (2.4), and the choice of $\epsilon$, for all $j=1, \ldots, k$, we have

$$
\begin{aligned}
d\left(Q_{j}, T_{C}\right)= & \mu\left(\left\{Q_{j} \neq T_{C}\right\}\right) \\
= & \mu\left(C \cap\left\{Q_{j} \neq T_{C}\right\}\right)+\mu\left(C^{\complement} \cap\left\{Q_{j} \neq T_{C}\right\}\right) \\
\leq & \mu\left(C \cap\left\{Q_{j} \neq f\left(z_{i}\right)\right\}\right)+\mu\left(C \cap\left\{f\left(z_{i}\right) \neq T\right\}\right) \\
& +\mu\left(C \cap\left\{T \neq T_{C}\right\}\right)+\mu\left(C^{\complement} \cap\left\{Q_{j} \neq 1\right\}\right) \\
\leq & \left(\mu(C) \mu\left(\left\{Q_{j} \neq f\left(z_{i}\right)\right\}\right)+2 \epsilon\right)+d\left(T, f\left(z_{i}\right)\right) \\
& +2 \epsilon+\left(\mu\left(C^{\complement}\right) \mu\left(\left\{Q_{j} \neq 1\right\}\right)+\epsilon\right) \\
\leq & \mu(C) d\left(Q_{j}, f\left(z_{i}\right)\right)+\mu\left(C^{\complement}\right) d\left(Q_{j}, 1\right)+6 \epsilon \\
< & \left(r_{j}-6 \epsilon\right)+6 \epsilon=r_{j} .
\end{aligned}
$$

Hence, $T_{C} \in \mathrm{B}_{r_{j}}\left(Q_{j}\right)$ for all $j$ and we are done.

This completes the proof of the theorem.

COROLlARY 2.5. Let $E$ be a countable, measure-preserving equivalence relation on $(X, \mu)$ which is not equality almost everywhere. Then $[E]$ is homeomorphic to $\ell^{2}$.

Proof. By the theorem of Dobrowolski and Toruńczyk cited above, it suffices to check that $[E]$ is not locally compact. This follows from the simple observation that every non-trivial full group contains an involution and the full group of any involution is isomorphic (as a topological group) to (MALG $\mu, \triangle$ ) which is easily verified to not be locally compact.

Remark. In fact, Bessaga and Pełczyński [1] have shown that $\mathrm{MALG}_{\mu}$ is homeomorphic to $\ell^{2}$. Corollary 2.5 can be considered a generalization of this result since if $T$ is a nontrivial involution, the full group $[T]$ is isomorphic to $\left(\mathrm{MALG}_{\mu}, \triangle\right)$.

\section{Automatic continuity}

The phenomenon of automatic continuity of group homomorphisms has recently been the subject of many investigations. Classical results state that Baire measurable homomorphisms between Polish groups (Pettis) and Haar measurable homomorphisms between locally compact groups (Weil with some countability assumptions and Kleppner [19] in full generality) are necessarily continuous but recently such results were obtained (for particular source groups) without any restrictions on the homomorphisms except that their target be separable. (The separability assumption for the target is always necessary; indeed, the identity map on a group is a homomorphism that is never continuous if the target is taken with the discrete topology and the source is non-discrete.) One way of thinking of this strong automatic continuity property is that the algebraic structure of the groups possessing it is so rigid that the axiom of choice is unable to produce pathological (non-continuous in the natural topology) homomorphisms. Kechris and Rosendal [17] showed that every homomorphism from a group with ample generics (including the group of permutations of the integers, the group of measure-preserving homeomorphisms of the Cantor space, and the group of Lipschitz homeomorphisms of the Baire space) to a separable group is continuous; Rosendal and Solecki [27] proved the same result for the 
homeomorphism group of the Cantor space, the group of order-preserving automorphisms of $\mathbf{Q}$, and the groups of orientation-preserving homeomorphisms of the real line and the circle; and Rosendal [26] proved automatic continuity for the homeomorphism groups of compact 2-manifolds. For details and further discussion, see those three papers.

Rosendal and Solecki [27] isolated a property that implies automatic continuity which we proceed to describe. Let $G$ be any topological group. A set $W \subseteq G$ is called countably syndetic if countably many left translates of $W$ cover $G$. Following [27], say that $G$ is Steinhaus if there exists a number $n$ such that for any symmetric, countably syndetic set $W, W^{n}$ contains an open neighborhood of the identity. It is proved in [27] that if $G$ is Steinhaus, then any homomorphism $f: G \rightarrow H$, where $H$ is an arbitrary separable topological group, is continuous.

THEOREM 3.1. Let E be an ergodic, measure-preserving, countable equivalence relation on the standard probability space $(X, \mu)$. Then the full group $[E]$ is Steinhaus. In particular, every homomorphism from $[E]$ to a separable group is continuous.

Proof. We borrow ideas and methods from [27]. Let $W \subseteq[E]$ be a symmetric, countably syndetic set and let $k_{1}, k_{2}, \ldots \in[E]$ be such that

$$
\bigcup_{n} k_{n} W=[E]
$$

For $B \in \mathrm{MALG}_{\mu}$, denote by $H_{B}$ the subgroup of $[E]$ consisting of the transformations whose support is contained in $B$. Say that a set $U \subseteq[E]$ is full for $B$ if

$$
\text { for all } T \in H_{B} \text { there exists } S \in U \text { such that }\left.T\right|_{B}=\left.S\right|_{B} \text {. }
$$

We use repeatedly and without reference the basic fact that because of the ergodicity of $E$, for all pairs of sets $A, B \in \mathrm{MALG}_{\mu}$ of the same measure, there exists an involution $T \in[E]$ with $T(A)=B$ and supp $T \subseteq A \cup B$ (see [16, Lemma 7.10]). The following lemma and its proof are similar to Claim 1 in the proof of Theorem 12 of [27].

Lemma 3.2. Let $\left\{B_{1}, B_{2}, \ldots\right\}$ be a collection of pairwise disjoint subsets of $X$. Then there exists $n \in \mathbf{N}$ such that $W^{2}$ is full for $B_{n}$.

Proof. It suffices to show that $k_{n} W$ is full for $B_{n}$ for some $n$ since then

$$
W^{2}=\left(k_{n} W\right)^{-1}\left(k_{n} W\right)
$$

is also full for $B_{n}$. Suppose that this is not the case. Then for each $n$, there exists $T_{n} \in H_{B_{n}}$ such that for all $S \in k_{n} W,\left.S\right|_{B_{n}} \neq\left. T_{n}\right|_{B_{n}}$. Define $T \in[E]$ by

$$
T x= \begin{cases}T_{n} x & \text { if } x \in B_{n}, \\ x & \text { if } x \notin \bigcup_{n} B_{n} .\end{cases}
$$

Then $T \notin k_{n} W$ for all $n$, contradicting (3.1).

LEMMA 3.3. There exists a non-empty $B \in \mathrm{MALG}_{\mu}$ such that $H_{B} \subseteq W^{36}$. 
Proof. Let $\left\{B_{1}, B_{2}, \ldots\right\}$ be any collection of pairwise disjoint non-empty subsets of $X$. By Lemma 3.2, there is $n_{0}$ such that $W^{2}$ is full for $B_{n_{0}}$. Set $B^{\prime}=B_{n_{0}}$. We show that $W^{2}$ contains a non-trivial involution whose support is contained in $B^{\prime}$. Indeed, let $T \in[E]$ be any involution with supp $T=B^{\prime}$. The group $[T]<[E]$ is uncountable and separable, hence there exists $n$ and $S_{1}, S_{2} \in[T]$ such that $0<d\left(S_{1}, S_{2}\right)<\mu\left(B^{\prime}\right) / 2$ and $S_{1}, S_{2} \in k_{n} W$. Set $S=S_{1} S_{2}$. Then

$$
S=S_{1} S_{2}=S_{1}^{-1} S_{2} \in\left(k_{n} W\right)^{-1}\left(k_{n} W\right)=W^{2}
$$

and

$$
\mu(\operatorname{supp} S)=d(1, S)=d\left(S_{1}, S_{2}\right)<\mu\left(B^{\prime}\right) / 2 .
$$

Note that every involution $U \in H_{B^{\prime}}$ with $\mu(\operatorname{supp} U)=\mu(\operatorname{supp} S)$ is conjugate to $S$ in $H_{B^{\prime}}$. Indeed, let $C \subseteq \operatorname{supp} S$ and $D \subseteq \operatorname{supp} U$ be such that $C \cup S(C)=\operatorname{supp} S, C \cap S(C)=\emptyset$, $D \cup S(D)=\operatorname{supp} U, D \cap U(D)=\emptyset$. Find $V_{1} \in H_{B^{\prime}}$ such that $V_{1}(C)=D$. Define $V_{2} \in H_{B^{\prime}}$ by

$$
V_{2} x= \begin{cases}V_{1} x & \text { if } x \in C, \\ U V_{1} S x & \text { if } x \in S(C)\end{cases}
$$

on supp $S$ and extend it arbitrarily to an element of $H_{B^{\prime}}$. Then it is easy to check that $V_{2} S V_{2}^{-1}=U$. By the fullness of $W^{2}$ for $B^{\prime}$, there exists $V_{3} \in W^{2}$ such that $\left.V_{3}\right|_{B^{\prime}}=V_{2}$. Then $U=V_{3} S V_{3}^{-1}$ and, thus, $U \in W^{6}$.

Let now $B \subseteq B^{\prime}$ be such that supp $S \subseteq B$ and $\mu(B)=2 \mu(\operatorname{supp} S)$ and note that every involution in $H_{B}$ can be written as a product $U_{1} U_{2}$ where $U_{1}, U_{2} \in H_{B^{\prime}}$ are involutions with $\mu\left(\operatorname{supp} U_{1}\right)=\mu\left(\operatorname{supp} U_{2}\right)=\mu(\operatorname{supp} S)$. Therefore, all involutions in $H_{B}$ are contained in $W^{12}$. Finally, by the argument in Ryzhikov [28], every element of $H_{B}$ is the product of three involutions, so $H_{B} \subseteq W^{36}$.

Now we are ready to complete the proof of the theorem. Fix $B$ as given by Lemma 3.3. Let $T_{1}, T_{2}, \ldots$ be a sequence of elements of $[E]$ with $\lim _{n \rightarrow \infty} T_{n}=1$. We show that some $T_{m}$ is in $W^{38}$, thus proving that $W^{38}$ contains an open neighborhood of the identity. Set $C_{n}=\operatorname{supp} T_{n}$. By passing to a subsequence, we can assume that $\sum_{n} \mu\left(C_{n}\right)<\mu(B)$. Let

$$
D=\bigcup_{n} k_{n}\left(C_{n}\right) .
$$

Fix $A \subseteq B$ with $\mu(A)=\mu(D)$. There exists $S^{\prime} \in[E]$ such that $S^{\prime}(A)=D$. By (3.1), there is $m$ such that $S^{\prime} \in k_{m} W$. If we set $S=k_{m}^{-1} S^{\prime}$, we have $S \in W$ and

$$
S(A)=k_{m}^{-1}(D) \supseteq C_{m} .
$$

Hence, $S^{-1} T_{m} S \in H_{B} \subseteq W^{36}$ and

$$
T_{m}=S\left(S^{-1} T_{m} S\right) S^{-1} \in W W^{36} W=W^{38},
$$

proving that $[E]$ is Steinhaus with exponent 38.

Remark. Note that the hypothesis of ergodicity cannot be omitted. If $T$ is a non-trivial involution, then the group $[T]$ is isomorphic to $\left(\mathrm{MALG}_{\mu}, \triangle\right)$ ) and the latter admits many different topologies. Indeed, $\mathrm{MALG}_{\mu}$ is a vector space over $\mathbf{Z} / 2 \mathbf{Z}$, hence has a Hamel basis, hence is isomorphic to any abelian group of exponent two and cardinality continuum (for example, $(\mathbf{Z} / 2 \mathbf{Z})^{\mathbf{N}}$ ). 


\section{Topological generators}

4.1. The hyperfinite case. In order to distinguish equivalence relations by the number of topological generators of their full groups, the first thing one has to show is that this number is not always infinite. We start with the simplest case, the equivalence relation generated by a single ergodic automorphism. In this subsection, $E$ will always be ergodic and hyperfinite. By Dye's theorem, there exists only one hyperfinite, ergodic equivalence relation (up to isomorphism), so we have the flexibility to consider any measure-preserving, ergodic automorphism as the generator of $E$. In order to produce a finitely generated dense subgroup of $[E]$, we use a specific minimal homeomorphism of the Cantor space as a topological model and the theory of topological full groups of minimal homeomorphisms, as developed by Giordano, Putnam and Skau [10]. In fact, our dense subgroup comes directly from an example of Matui [20].

We recall some concepts and definitions from [10]. Let $\phi$ be an aperiodic (with all of its orbits infinite) homeomorphism of the Cantor space $X$. For every homeomorphism $\gamma$ of $X$ preserving the orbits of $\phi$, define its associated cocycle $n_{\gamma}: X \rightarrow \mathbf{Z}$, where $\mathbf{Z}$ denotes the discrete group of the integers, by

$$
n_{\gamma}(x)=n \Longleftrightarrow \gamma(x)=\phi^{n}(x) .
$$

Define the topological full group of $\phi$ by

$$
[[\phi]]=\left\{\gamma \in \operatorname{Homeo}(X): \forall x \exists n \in \mathbf{Z} \gamma(x)=\phi^{n}(x) \text { and } n_{\gamma} \text { is continuous }\right\} .
$$

Since there are only countably many continuous functions $X \rightarrow \mathbf{Z}$, the group [[ $\phi]]$ is always countable. It is a remarkable fact, discovered by Matui, that those groups are sometimes finitely generated. We will prove that topological full groups of minimal homeomorphisms are dense in the corresponding measure-theoretic full groups (with respect to an invariant measure), and thus provide a rich supply of finitely generated dense subgroups of $[E]$.

Let $\phi$ be a minimal homeomorphism of the Cantor space $X$ and $\mu$ be any $\phi$-invariant, Borel, probability measure on $X$. Define the index map $I:[[\phi]] \rightarrow \mathbf{R}$ by

$$
I(\gamma)=\int n_{\gamma} d \mu
$$

It turns out to be a homomorphism $[[\phi]] \rightarrow \mathbf{Z}$ which does not depend on the choice of $\mu$. Its kernel is denoted by $[[\phi]]_{0}$. For all of this, see $[\mathbf{1 0}]$.

Proposition 4.1. Let $\phi$ be a minimal homeomorphism of the Cantor space $X$ and $\mu$ be an invariant measure. Let $E$ be the equivalence relation induced by $\phi$. Then the countable group $[[\phi]]_{0}$ is dense in $[E]$.

Proof. By [15, Proposition 3.7], it is sufficient to show that given pairwise disjoint clopen subsets $A_{1}, \ldots, A_{k} \subseteq X$, integers $n_{1}, \ldots, n_{k}$ such that the sets $\phi^{n_{1}}\left(A_{1}\right), \ldots, \phi^{n_{k}}\left(A_{k}\right)$ are pairwise disjoint, and $\epsilon>0$, we can produce $\psi \in[[\phi]]_{0}$ with the property

$$
\mu\left(\left\{\psi \neq \phi^{n_{i}}\right\} \cap A_{i}\right)<\epsilon \quad \text { for all } i=1, \ldots, k .
$$

Let $N_{0}$ be an integer so big that $\left|n_{i}\right| / N_{0}<\epsilon$ for all $i$. Let $D \subseteq X$ be clopen with

$$
D, \phi(D), \ldots, \phi^{N_{0}-1}(D) \text { disjoint. }
$$


Build a Kakutani-Rokhlin stack with base $D$ compatible with the sets $A_{i}, \phi^{n_{i}}\left(A_{i}\right)$, $i=1, \ldots, k$, i.e. find numbers $m \in \mathbf{N}$ (the number of towers in the stack) and $J(1), \ldots, J(m) \in \mathbf{N}$ (the heights of the towers), and a clopen partition

$$
\{Z(l, j): l=1, \ldots, m ; j=0, \ldots, J(l)-1\}
$$

of $X$ satisfying the conditions:

- $\phi(Z(l, j))=Z(l, j+1), j=0, \ldots, J(l)-2$;

- $\phi\left(\bigcup_{l=1}^{m} Z(l, J(l)-1)\right)=\bigcup_{l=1}^{m} Z(l, 0)$;

- $\bigcup_{l=1}^{m} Z(l, 0)=D$;

- $\quad$ each one of the sets $A_{i}, \phi^{n_{i}}\left(A_{i}\right)(i=0, \ldots, k-1)$ is the union of some elements of the partition.

For details on how to achieve this, see Herman, Putnam and Skau [11, Lemma 4.1]. By (4.2), the height of each tower in the stack is at least $N_{0}$. Let $\sigma_{l}^{\prime}$ be the partial function $J(l) \rightarrow J(l)$ (as is customary, we identify $J(l)$ with the set $\{0,1, \ldots, J(l)-1\}$ ) defined by

$$
\sigma_{l}^{\prime}(a)=b \Longleftrightarrow \text { there exists } i \text { such that } Z(l, a) \subseteq A_{i} \text { and } b=a+n_{i} .
$$

Since the collections $\left\{A_{i}\right\}_{i<k}$ and $\left\{\phi^{n_{i}}\left(A_{i}\right)\right\}_{i<k}$ are each pairwise disjoint, $\sigma_{l}^{\prime}$ is injective, so it extends to a permutation $\sigma_{l} \in S_{J(l)}$. Finally, define

$$
\psi(x)=\phi^{n}(x) \Longleftrightarrow x \in Z(l, j) \text { and } n=\sigma_{l}(j)-j .
$$

For the value of the index homomorphism, we have the following simple computation (using that $\mu$ is invariant under $\phi$ and, hence, $\mu\left(Z\left(l, j_{1}\right)\right)=\mu\left(Z\left(l, j_{2}\right)\right)$ for $\left.j_{1}, j_{2}<J(l)\right)$ :

$$
I(\psi)=\int n_{\psi} d \mu=\sum_{l=1}^{m} \mu(Z(l, 0)) \sum_{j=0}^{J(l)-1}\left(\sigma_{l}(j)-j\right)=0 .
$$

On each of the $A_{i}, \psi$ agrees with $\phi^{n_{i}}$ everywhere except possibly on the set

$$
U_{i}=\bigcup_{l} \bigcup_{j \in C_{l, i}} Z(l, j)
$$

where $C_{l, i}=\left\{0, \ldots, 1-n_{i}\right\}$ if $n_{i}<0$ and $C_{l, i}=\left\{J(l)-n_{i}, \ldots, J(l)-1\right\}$ if $n_{i} \geq 0$. However,

$$
\mu\left(U_{i}\right)=\sum_{l=1}^{m} \frac{\left|n_{i}\right|}{J(l)} \mu\left(\bigcup_{j<J(l)} Z(l, j)\right) \leq \frac{\left|n_{i}\right|}{N_{0}}<\epsilon,
$$

so we are done.

Remark. A result similar to Proposition 4.1 was also independently proved by Medynets [21]. More generally, Miller [23] has shown that if $X$ is a zero-dimensional Polish space, $\Gamma$ is a countable group acting on $X$ by homeomorphisms, and $\mu$ is a Borel probability measure on $X$, then the topological full group $[[\Gamma]]$ (defined by a formula analogous to (4.1)) is dense in the full group $\left[E_{\Gamma}^{X}\right]_{\mu}$. Hjorth and Molberg [12] have shown that every countable group admits a free action on the Cantor space with an invariant probability measure, so it seems that results of this type could be applied in rather general situations. Unfortunately, however, little is currently known about topological full groups that arise from actions by homeomorphisms of non-amenable groups. 
Matui [20] has recently characterized the minimal homeomorphisms $\phi$ for which the group $[[\phi]]$ (or, equivalently, the group $\left[\left[\phi_{0}\right]\right]$ ) is finitely generated. He proved that $[[\phi]]$ is finitely generated if and only if $\phi$ is conjugate to a minimal subshift and satisfies an additional technical condition. He also calculated in a few examples specific generators for those groups. Currently, there are examples of minimal homeomorphisms whose topological full groups have three generators [20, Examples 6.1 and 6.2]. It is not known whether such topological full groups with two generators exist.

Thus, by Proposition 4.1, we have the following theorem which answers a question of Kechris.

THEOREM 4.2. Let $E$ be an ergodic, hyperfinite equivalence relation. Then $t([E]) \leq 3$.

Since $[E]$ is not abelian, we must have $t([E]) \geq 2$. However, we do not know what the exact value of $t([E])$ is.

Question 4.3. Let $E$ be ergodic and hyperfinite. Is $t([E])$ equal to two or three?

It would also be interesting to find an elementary construction of a dense subgroup of $[E]$ (for a hyperfinite $E$ ) with few generators for some concrete realization of $E$. (The examples of Matui are concrete enough but his computations of the generators rely heavily on the $C^{*}$-algebraic machinery of Giordano, Putnam and Skau.) In this direction, Kittrell [18], using $E_{0}$ (the equivalence relation on $(\mathbf{Z} / 2 \mathbf{Z})^{\mathbf{N}}$ generated by the action of the subgroup $(\mathbf{Z} / 2 \mathbf{Z})^{<\mathbf{N}}$ by translation) as a model and purely combinatorial techniques, has found 18 generators for a dense subgroup of $[E]$.

4.2. The general case. Theorem 4.2 provides an example of a situation where $[E]$ is topologically finitely generated. In the remaining part of this section, we develop techniques to characterize exactly when this happens. The following proposition is the main tool we use.

If $G$ is a group and $A \subseteq G$ a subset, denote by $\langle A\rangle$ the subgroup of $G$ generated by $A$.

Proposition 4.4. Let $(X, \mu)$ be a standard probability space and $T_{0}, T_{1}, \ldots \in$ $\operatorname{Aut}(X, \mu)$ be involutions. Let $E$ be the equivalence relation generated by them. Then $\left\langle\bigcup_{j \in \mathbf{N}}\left[T_{j}\right]\right\rangle$ is dense in $[E]$.

Proof. Since [E] is generated by involutions [5] (see also Ryzhikov [28]; in fact, the results in these papers are stated for the full automorphism group $\operatorname{Aut}(X, \mu)$ but one readily sees that the proofs are also valid for full groups), it suffices to approximate involutions. Fix $I \in[E], I^{2}=1$. For $T \in \operatorname{Aut}(X, \mu)$ and $A \subseteq X$ such that $A \cap T(A)=\emptyset$, define $T^{A} \in[T]$ by

$$
T^{A} x= \begin{cases}T x & \text { if } x \in A, \\ T^{-1} x & \text { if } x \in T(A), \\ x & \text { otherwise. }\end{cases}
$$

Lemma 4.5. Let $T_{1}, \ldots, T_{n} \in \operatorname{Aut}(X, \mu)$ and set $S_{k}=T_{k} T_{k-1} \cdots T_{1}$ for $k=1, \ldots, n$. Let $A_{0} \subseteq X$ be Borel and define $A_{k}=S_{k}\left(A_{0}\right)$ for $k=1, \ldots, n$. Suppose that $A_{0}, A_{1}, \ldots, A_{n}$ are pairwise disjoint. Then $S_{n}^{A_{0}} \in\left\langle T_{1}^{A_{0}}, T_{2}^{A_{1}}, \ldots, T_{n}^{A_{n-1}}\right\rangle$. 


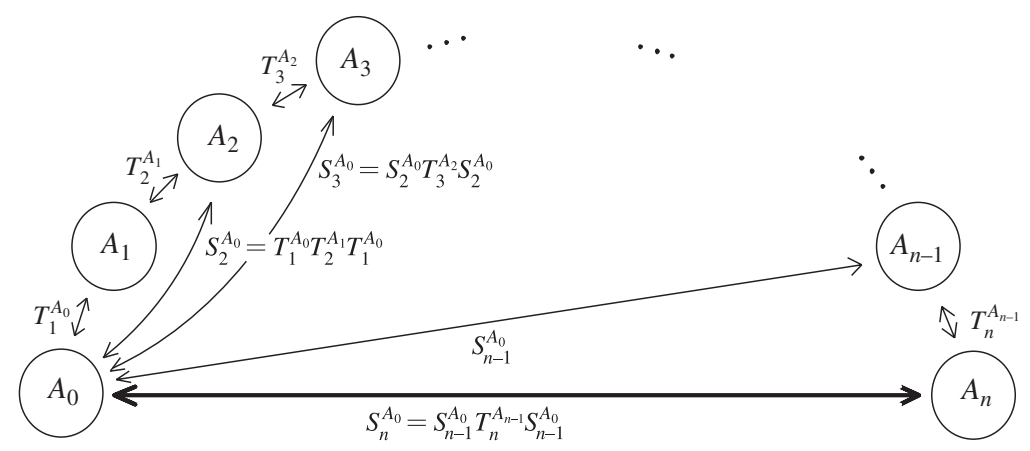

FIGURE 1. Building $S_{n}^{A_{0}}$ out of $T_{1}^{A_{0}}, T_{2}^{A_{1}}, \ldots, T_{n}^{A_{n-1}}$.

Proof. We have $S_{1}^{A_{0}}=T_{1}^{A_{0}}$ by definition. It will then be enough to show that

$$
S_{k+1}^{A_{0}}=S_{k}^{A_{0}} T_{k+1}^{A_{k}} S_{k}^{A_{0}} \quad \text { for } k=1, \ldots, n-1 .
$$

This can best be seen from the diagram in Figure 1.

Lemma 4.6. Let $T \in \operatorname{Aut}(X, \mu)$ be an involution and $\left\{X_{n}\right\}_{n \in \mathbf{N}}$ be a Borel partition of $X$. Let

$$
\Phi=\left\{T^{A}: A \cap T(A)=\emptyset \text { and } A \subseteq X_{n} \text { for some } n\right\} .
$$

Then $T \in \overline{\langle\Phi\rangle}$.

Proof. Without loss of generality, we can assume that $\operatorname{supp} T=X$. By refining the partition if necessary, we can assume that it is $T$-invariant and, for each $n, T\left(X_{n}\right) \cap$ $X_{n}=\emptyset$. Let $\left\{X_{n_{i}}\right\}$ be a transversal for the action of $T$ on the partition $\left\{X_{n}\right\}$, i.e. $\bigcup_{i} X_{n_{i}} \cup T\left(X_{n_{i}}\right)=X$ and $X_{n_{i}} \cap T\left(X_{n_{j}}\right)=\emptyset$. Then $T=\prod_{i=0}^{\infty} T^{X_{n_{i}}}$ and since, for each

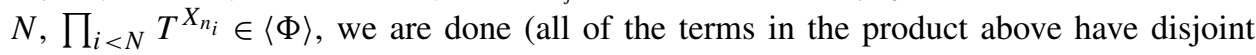
supports which implies that the finite products converge to the infinite product).

In order to prove Proposition 4.4, we first use Lemma 4.5 to show that we can approximate the automorphisms $I^{A}$ for certain sets $A$ and then glue them together using Lemma 4.6 in order to approximate $I$.

Let $\mathcal{G}=\bigcup_{j \in \mathbf{N}}$ graph $T_{j}$. We can view $\mathcal{G}$ as a Borel graph on $X$ whose connected components are the $E$-equivalence classes. Label the edge $(x, y)$ of $\mathcal{G}$ with $j$ if $T_{j} x=y$ (some edges may have more than one label). For $s \in \mathbf{N}^{<\mathbf{N}}$, denote by $|s|$ the length of $s$. For each $x \in X$, let $s_{x} \in \mathbf{N}^{<\mathbf{N}}$ be the sequence of labels of the lexicographically least among the shortest $\mathcal{G}$-paths from $x$ to $I(x)$ so that

$$
T_{S_{x}\left(\left|s_{x}\right|-1\right)} T_{S_{x}\left(\left|s_{x}\right|-2\right)} \cdots T_{S_{x}(0)}(x)=I(x) .
$$

For $x \in X$ and $k \leq\left|s_{x}\right|$, set

$$
J_{k}(x)=T_{s_{x}(k-1)} T_{s_{x}(k-2)} \cdots T_{s_{x}(0)}(x) .
$$

(Thus, id $=J_{0}, J_{1}, J_{2}, \ldots$ are partial automorphisms of $E$.) By the choice of $s_{x}$,

$$
\text { the points } x, J_{1}(x), \ldots, J_{\left|s_{x}\right|}(x) \text { are distinct. }
$$

\section{CAMBRIDGE JDURNALS}


The mapping $x \mapsto s_{x}$ is clearly Borel. For each $s \in \mathbf{N}^{<\mathbf{N}}$, let

$$
X_{s}=\left\{x \in X: s_{x}=s\right\} .
$$

By Lemma 4.6, in order to approximate $I$, it suffices to approximate $I^{A}$ for sets $A$ for which $A \cap I(A)=\emptyset$ and $A \subseteq X_{s}$ for some $s$. Fix such $A$ and $s$. Let $\mathcal{B}$ be a countable dense subalgebra of $\mathrm{MALG}_{\mu}$. By (4.3), for each $x \in A$, there exist pairwise disjoint $U_{0}, \ldots, U_{|s|} \in \mathcal{B}$ such that $J_{i}(x) \in U_{i}$ for all $i \leq|s|$. Let $\mathcal{A}$ be the countable set of all sequences $\alpha=\left(U_{0}, U_{1}, \ldots, U_{|s|}\right)$ of pairwise disjoint elements of $\mathcal{B}$ of length $|s|+1$. For $\alpha \in \mathcal{A}$, let

$$
A_{\alpha}=\left\{x \in A: \forall i \leq|s| J_{i}(x) \in \alpha(i)\right\} .
$$

Thus, $\bigcup_{\alpha \in \mathcal{A}} A_{\alpha}=A$. Let $\left\{\alpha_{n}\right\}_{n \in \mathbf{N}}$ be an enumeration of $\mathcal{A}$ and inductively define

$$
B_{n}=A_{\alpha_{n}} \backslash \bigcup_{j<n} B_{j} .
$$

Then $\left\{B_{0}, B_{1}, \ldots\right\}$ is a partition of $A$ and for each $n$, the sets $B_{n}, J_{1}\left(B_{n}\right), \ldots, J_{|s|}\left(B_{n}\right)$ are pairwise disjoint (since $J_{i}\left(A_{\alpha}\right) \subseteq \alpha(i)$ by the definition of $A_{\alpha}$ ). By Lemma 4.5, $I^{B_{n}} \in\left\langle\bigcup_{j \in \mathbf{N}}\left[T_{j}\right]\right\rangle$ for all $n$ and applying Lemma 4.6 again shows that $I^{A} \in \overline{\left\langle\bigcup_{j \in \mathbf{N}}\left[T_{j}\right]\right\rangle}$.

Recall that if $\left\{E_{1}, E_{2}, \ldots\right\}$ is a countable collection of equivalence relations, their join, denoted by $E_{1} \vee E_{2} \vee \cdots$, is the smallest equivalence relation that contains all of them. Since every equivalence relation is generated by involutions, Proposition 4.4 generalizes to the following.

THEOREM 4.7. Let $E_{1}, E_{2}, \ldots$ be countable, measure-preserving equivalence relations on $(X, \mu)$ and $E$ be their join. Then $\left\langle\bigcup_{n \in \mathbf{N}}\left[E_{n}\right]\right\rangle$ is dense in $[E]$.

In order to continue our analysis, we need the notion of cost of an equivalence relation introduced by Levitt and further developed by Gaboriau. We briefly recall the definition and refer the reader to [16] for more details. If $E$ is an equivalence relation, we denote by $[[E]]$ the set of all partial automorphisms of $E$, i.e. all partial Borel bijections of $X$ whose graphs are contained in $E$. Since $E$ is measure-preserving, for all $\psi \in[[E]]$, $\mu(\operatorname{dom} \psi)=\mu(\operatorname{rng} \psi)$. An $L$-graphing of an equivalence relation $E$ is a countable subset $\Psi \subseteq[[E]]$ such that $E$ is the smallest equivalence relation containing the graphs of all elements of $\Psi$. The cost of $\Psi$ is defined as

$$
\operatorname{cost} \Psi=\sum_{\psi \in \Psi} \mu(\operatorname{dom} \psi)
$$

and the cost of $E$ is given by

$$
\operatorname{cost} E=\inf \{\operatorname{cost} \Psi: \Psi \text { is an L-graphing of } E\} .
$$

The cost can be finite or infinite and if $E$ is ergodic, cost $E \geq 1$. One of the main results of Gaboriau's theory [7] is that if $E$ is generated by a free, ergodic action of $\mathbf{F}_{n}$, then $\operatorname{cost} E=n$, i.e. the L-graphing given by the group generators is optimal in this case.

The following lemma was proved by Ben Miller. 
LEMMA 4.8. Let $E$ be an ergodic equivalence relation of cost less than $n$. Then there exist finite equivalence relations $F_{1}, \ldots, F_{n}$ such that $F_{1} \vee \cdots \vee F_{n}=E$.

Proof. Since $E$ is ergodic, cost $E \geq 1$ and, hence, $n \geq 2$. By [16, Lemma 27.7] and its proof, there exist $\phi_{1}, \ldots, \phi_{n-1} \in[E]$ and $\psi \in[[E]]$ with $\mu(\operatorname{dom} \psi)<1$ such that $\phi_{1}, \ldots, \phi_{n-1}, \psi$ generate $E$ and $\phi_{1}$ is ergodic. Let $E_{1}, \ldots, E_{n-1}$ denote the orbit equivalence relations of $\phi_{1}, \ldots, \phi_{n-1}$, respectively. We will build $F_{1}, \ldots, F_{n-1}$ as finite approximations of $E_{1}, \ldots, E_{n-1}$ and use $F_{n}$ to glue the pieces together.

Without loss of generality, we can assume that $\phi_{2}, \ldots, \phi_{n-1}$ are aperiodic. (If, say, $\phi_{2}$ is periodic on the positive set $D$, we can set $F_{2}$ to be equal to $E_{\phi_{2}}^{D}$ on $D$ and proceed with the aperiodic part exactly as below.) Set $B=\operatorname{dom} \psi$ and let $\epsilon<(1-\mu(B)) / 2 n$. Let $A_{1}, \ldots, A_{n-1}$ be complete sections for $E_{1}, \ldots, E_{n-1}$ such that $\mu\left(A_{i}\right)<\epsilon, A_{i} \cap$ $\phi_{i}\left(A_{i}\right)=\emptyset$ for $i=1, \ldots, n-1$, and $\left(A_{1} \cup \phi_{1}\left(A_{1}\right)\right) \cap B=\emptyset$. Since $\phi_{1}, \ldots, \phi_{n-1}$ are aperiodic, $A_{i}$ is $\phi_{i}$-birecurrent. For each $i=1, \ldots, n-1$, define the finite equivalence relation $F_{i}$ by

$$
x F_{i} y \Longleftrightarrow \exists n \in \mathbf{Z} \phi_{i}^{n}(x)=y \text { and } \forall k \in(0, n] \cup(n, 0] \phi_{i}^{k}(x) \notin A_{i},
$$

i.e. $F_{i}$ is given by splitting the orbits of $\phi_{i}$ into finite pieces using the complete section $A_{i}$. Define $\xi_{i} \in[[E]]$ to be the involution $\left.\left.\phi_{i}\right|_{A_{i}} \cup \phi_{i}^{-1}\right|_{\phi_{i}\left(A_{i}\right)}$. Note that the equivalence relation generated by $F_{i}$ and $\xi_{i}$ is $E_{i}$. Set $B_{1}=\operatorname{dom} \xi_{1}$ and let $B_{2}, B_{3}, \ldots, B_{n-1}$ be disjoint subsets of $X \backslash\left(B \cup B_{1}\right)$ such that $\mu\left(B_{i}\right)=\mu\left(\operatorname{dom} \xi_{i}\right)=2 \mu\left(A_{i}\right)$. Let $\theta^{\prime} \in\left[E_{1}\right]$ be such that $\theta^{\prime}(\operatorname{rng} \psi)=B$ and let $\theta_{i} \in\left[E_{1}\right]$ be such that $\theta_{i}\left(\operatorname{dom} \xi_{i}\right)=B_{i}$ for $i=2, \ldots, n-1$. Define $\psi^{\prime}=\theta^{\prime} \psi$ and $\eta_{i}=\theta_{i} \xi_{i} \theta_{i}^{-1}$ for $i=2, \ldots, n-1$. Note that $\psi^{\prime}$ is an automorphism of $B$. Again, without loss of generality, we can assume that $\psi^{\prime}$ is aperiodic. Let $C$ be a complete section for $E_{\psi^{\prime}}^{B}$ such that $\mu(C)<\epsilon$ and $C \cap \psi^{\prime}(C)=\emptyset$. Let the finite equivalence relation $F_{n}^{\prime}$ on $B$ be the splitting of the orbits of $\psi^{\prime}$ into finite pieces using the complete section $C$ (defined by a formula similar to (4.4)). Define $\xi_{0} \in[[E]]$ to be the involution $\left.\left.\psi^{\prime}\right|_{C} \cup \psi^{\prime-1}\right|_{\psi^{\prime}(C)}$. Let $B_{0}$ be a set of measure $\mu\left(\operatorname{dom} \xi_{0}\right)$ disjoint from $B \cup B_{1} \cup B_{2} \cup \cdots \cup B_{n-1}$. Let $\theta_{0} \in\left[E_{1}\right]$ be such that $\theta_{0}$ (dom $\left.\xi_{0}\right)=B_{0}$ and define $\eta_{0}=\theta_{0} \xi_{0} \theta_{0}^{-1}$. Finally, define $F_{n}$ by

$$
F_{n}=\left.F_{n}^{\prime} \cup E_{\eta_{0}}^{B_{0}} \cup E_{\eta_{1}}^{B_{1}} \cup \cdots \cup E_{\eta_{n-1}}^{B_{n-1}} \cup \mathrm{id}\right|_{X \backslash\left(B \cup B_{0} \cup B_{1} \cup \cdots \cup B_{n-1}\right)} .
$$

Now it is easy to see that $E_{i} \subseteq E_{1} \vee F_{i} \subseteq F_{1} \vee F_{n} \vee F_{i}$ for $i=1, \ldots, n-1$ and $E_{\psi}^{X} \subseteq$ $E_{1} \vee F_{n} \subseteq F_{1} \vee F_{n}$, showing that $F_{1} \vee \cdots \vee F_{n}=E$.

LEMMA 4.9. Let $F \subseteq E$ be equivalence relations on $(X, \mu)$ where $F$ is finite and $E$ is ergodic. Then there exists an ergodic, hyperfinite equivalence relation $E^{\prime}$ such that $F \subseteq E^{\prime} \subseteq E$.

Proof. Since $F$ is finite, the space $Y=X / F$ is standard Borel. Let $\pi: X \rightarrow Y$ be the canonical projection. Set $v=\pi_{*} \mu$ and define the equivalence relation $E / F$ on $Y$ by

$y_{1} E / F y_{2} \Longleftrightarrow$ there exist $x_{1}, x_{2} \in X$ such that $x_{1} E x_{2}$ and $\pi\left(x_{1}\right)=y_{1}$ and $\pi\left(x_{2}\right)=y_{2}$.

Since $\mu$ is non-atomic, $v$ is non-atomic and since $E$ is $\mu$-ergodic, $E / F$ is v-ergodic. Pick any ergodic $T \in[E / F]$ (such a $T$ exists by [15, Theorem 3.5]) and let $F^{\prime}$ be the 
equivalence relation on $Y$ generated by $T$. Finally, let $E^{\prime}=\pi^{-1}\left(F^{\prime}\right)$. We check that this $E^{\prime}$ works.

The inclusions $F \subseteq E^{\prime} \subseteq E$ are obvious. Next, the ergodicity of $E^{\prime}$ follows from the ergodicity of $F^{\prime}$. Finally, write $F^{\prime}=\bigcup_{n} F_{n}^{\prime}$ as the increasing union of finite equivalence relations on $Y$. Let for each $n, E_{n}^{\prime}=\pi^{-1}\left(F_{n}^{\prime}\right)$. Since $F$ is finite, all of the $E_{n}^{\prime}$ are finite. Also, $E^{\prime}=\bigcup_{n} E_{n}^{\prime}$ and the union is clearly increasing, so $E^{\prime}$ is hyperfinite.

THEOREM 4.10. Let $E$ be an ergodic equivalence relation with $\operatorname{cost} E<n$ for some $n \in \mathbf{N}$. Then $t([E]) \leq 3 n$.

Proof. By Lemma 4.8, there exist finite equivalence relations $F_{1}, \ldots, F_{n}$ such that $\bigvee_{i=1}^{n} F_{i}=E$. Use Lemma 4.9 to find, for each $i \leq n$, an ergodic, hyperfinite equivalence relation $E_{i}$ such that $F_{i} \subseteq E_{i} \subseteq E$. Then, $E=\bigvee_{i} E_{i}$ and applying Theorems 4.2 and 4.7, we obtain the desired upper bound.

COROLlaRY 4.11. Let $E$ be an ergodic equivalence relation on $(X, \mu)$. Then the following are equivalent:

(i) E can be generated by an action of a finitely generated group;

(ii) E has finite cost;

(iii) $[E]$ is topologically finitely generated.

Proof. The implication (i) $\Rightarrow$ (ii) is obvious and (ii) $\Rightarrow$ (i) was proved by Hjorth and Kechris (see [16, Lemma 27.7]). The implication (iii) $\Rightarrow$ (i) is also clear (as every group dense in $[E]$ generates $E$ ) and, finally, (ii) $\Rightarrow$ (iii) follows from Theorem 4.10 .

For free actions of free groups, the theory of cost allows us to obtain a lower bound for $t([E])$ as well.

COROLlary 4.12. Let $E$ be generated by a free, ergodic action of $\mathbf{F}_{n}$. Then

$$
n+1 \leq t([E]) \leq 3(n+1) .
$$

Proof. To prove the lower bound, suppose, towards a contradiction, that there is a set of automorphisms $\Phi \subseteq[E],|\Phi|=n$ with $\langle\Phi\rangle$ dense in $[E]$. Since $E=E_{\Phi}^{X}$ has cost $n, \Phi$ must act freely. Indeed, $\Phi$, considered as an L-graphing, realizes the cost of $E$, hence, by [16, Proposition 19.1], it is a treeing, hence the action is free. Therefore,

$$
\langle\Phi\rangle \subseteq\{T \in[E]: d(1, T)=1\} \cup\{1\}
$$

which is a closed, nowhere dense set in $[E]$ : a contradiction.

The upper bound follows from Theorem 4.10.

This corollary provides the first topological group distinction between the full groups of equivalence relations generated by free, ergodic actions of $\mathbf{F}_{m}$ and $\mathbf{F}_{n}$, at least when $m$ and $n$ are sufficiently far apart.

It would be interesting to improve those bounds. For example, if an action $\mathbf{F}_{n} \curvearrowright X$ is mixing and $\mathbf{F}_{n}=\left\langle\gamma_{1}, \ldots, \gamma_{n}\right\rangle$, then every $E_{\gamma_{i}}^{X}$ is hyperfinite and ergodic, so applying Theorems 4.2 and 4.7 yields the upper bound $t\left(\left[E_{\mathbf{F}_{n}}^{X}\right]\right) \leq 3 n$. 
Question 4.13. Let $E$ be generated by a free, ergodic action of $\mathbf{F}_{n}$. Is the number $t([E])$ independent of the action? If yes, what is it?

On another note, Proposition 4.4 allows us to associate with each uniformly closed, separable group $G \leq \operatorname{Aut}(X, \mu)$ a largest equivalence relation $F_{G}$ such that $\left[F_{G}\right] \leq G$.

Proposition 4.14. Suppose that $G \leq \operatorname{Aut}(X, \mu)$ is uniformly closed and separable. Then there is a largest countable equivalence relation $F_{G}$ such that its full group is contained in G. Moreover, $\left[F_{G}\right]$ is normal in $G$.

Proof. Let $\mathcal{F}$ be a maximal family of involutions whose full groups are contained in $G$ and which are almost everywhere different on their supports, i.e.

$$
\text { for all } T, S \in \mathcal{F} \quad \mu(\{x: T x=S x \neq x\})>0 \Longrightarrow T=S .
$$

Since $G$ is separable, $\mathcal{F}$ must be countable. Indeed, if $\mathcal{F}$ is uncountable, there exist an uncountable $\mathcal{A} \subseteq \mathcal{F}$ and $\epsilon>0$ such that for all $T \in \mathcal{A}, \mu(\operatorname{supp} T)>\epsilon$. Then for all $T, S \in \mathcal{A}, d(T, S)>2 \epsilon$, contradicting the separability of $G$. Now let $F_{G}=E_{\mathcal{F}}^{X}$. Proposition 4.4 and the fact that $G$ is closed imply that $\left[F_{G}\right] \leq G$.

Suppose now that $[E] \leq G$ for some equivalence relation $E$ but $[E] \not \leq\left[F_{G}\right]$. Then there exists an involution $T \in[E]$ such that $T \notin\left[F_{G}\right]$. Thus, there is a non-null $T$-invariant set $A \subseteq \operatorname{supp} T$ such that

$$
\text { for all } x \in A, S \in \mathcal{F} \quad T x \neq S x \text {. }
$$

Now set $T^{\prime}=\left.\left.T\right|_{A} \cup \mathrm{id}\right|_{A^{\mathrm{C}}}$. It is clear that $\left[T^{\prime}\right] \leq[E] \leq G$ and $T^{\prime}$ is everywhere different on its support from the elements of $\mathcal{F}$, contradicting the maximality of $\mathcal{F}$.

Finally, normality is clear since the property of being a full group is preserved under conjugation by elements of $\operatorname{Aut}(X, \mu)$.

Acknowledgements. We would like to thank our respective advisors G. Hjorth and A. S. Kechris for encouragement, support and guidance as well as B. Miller for the proof of Lemma 4.8 and useful discussions. We are also grateful to the referee for making useful comments and providing some references. The second author was partially supported by NSF grant DMS-0455285.

\section{REFERENCES}

[1] C. Bessaga and A. Pełczyński. Selected Topics in Infinite-dimensional Topology. PWN-Polish Scientific Publishers, Warsaw, 1975.

[2] A. I. Danilenko. The topological structure of Polish groups and groupoids of measure space transformations. Publ. Res. Inst. Math. Sci. 31(5) (1995), 913-940.

[3] T. Dobrowolski and H. Toruńczyk. Separable complete ANRs admitting a group structure are Hilbert manifolds. Topology Appl. 12(3) (1981), 229-235.

[4] H. A. Dye. On groups of measure preserving transformations. II. Amer. J. Math. 85 (1963), 551-576.

[5] A. Fathi. Le groupe des transformations de $[0,1]$ qui préservent la mesure de Lebesgue est un groupe simple. Israel J. Math. 29(2-3) (1978), 302-308. 
[6] J. Feldman and C. C. Moore. Ergodic equivalence relations, cohomology, and von Neumann algebras. I. Trans. Amer. Math. Soc. 234(2) (1977), 289-324.

[7] D. Gaboriau. Coût des relations d'équivalence et des groupes. Invent. Math. 139(1) (2000), 41-98.

[8] D. Gaboriau and S. Popa. An uncountable family of nonorbit equivalent actions of $\mathbb{F}_{n}$. J. Amer. Math. Soc. 18(3) (2005), 547-559 (electronic).

[9] T. Giordano and V. Pestov. Some extremely amenable groups. C. R. Math. Acad. Sci. Paris 334(4) (2002), 273-278.

[10] T. Giordano, I. F. Putnam and C. F. Skau. Full groups of Cantor minimal systems. Israel J. Math. 111 (1999), 285-320.

[11] R. H. Herman, I. F. Putnam and C. F. Skau. Ordered Bratteli diagrams, dimension groups and topological dynamics. Internat. J. Math. 3(6) (1992), 827-864.

[12] G. Hjorth and M. Molberg. Free continuous actions on zero-dimensional spaces. Topology Appl. 153(7) (2006), 1116-1131.

[13] A. Ioana. Orbit inequivalent actions for groups containing a copy of $\mathbb{F}_{2}$. Preprint, available at arXiv:math/0701027, 2007.

[14] M. Keane. Contractibility of the automorphism group of a nonatomic measure space. Proc. Amer. Math. Soc. 26 (1970), 420-422.

[15] A. S. Kechris. Global aspects of ergodic group actions and equivalence relations. Available at http://www.math.caltech.edu/people/kechris.html.

[16] A. S. Kechris and B. D. Miller. Topics in Orbit Equivalence (Lecture Notes in Mathematics, 1852). Springer, Berlin, 2004.

[17] A. S. Kechris and C. Rosendal. Turbulence, amalgamation and generic automorphisms of homogeneous structures. Proc. Lond. Math. Soc. 94(2) (2007), 302-350.

[18] J. Kittrell. Full groups and hyperfiniteness. PhD Thesis, UCLA, 2007.

[19] A. Kleppner. Measurable homomorphisms of locally compact groups. Proc. Amer. Math. Soc. 106(2) (1989), 391-395.

[20] H. Matui. Some remarks on topological full groups of Cantor minimal systems. Internat. J. Math. 17(2) (2006), 231-251.

[21] K. Medynets. On approximation of homeomorphisms of a Cantor set. Fund. Math. 194(1) (2007), 1-13.

[22] J. Melleray. Topology of the isometry group of the Urysohn space. Unpublished note available at http://math.univ-lyon1.fr/ melleray/.

[23] B. D. Miller. Density of topological full groups. Unpublished note available at http://glimmeffros.googlepages.com/, 2005.

[24] N. T. Nhu. The group of measure preserving transformations of the unit interval is an absolute retract. Proc. Amer. Math. Soc. 110(2) (1990), 515-522.

[25] V. Pestov. Dynamics of Infinite-dimensional Groups (University Lecture Series, 40). American Mathematical Society, Providence, RI, 2006 (The Ramsey-Dvoretzky-Milman phenomenon, Revised edition of Dynamics of infinite-dimensional groups and Ramsey-type phenomena, Inst. Mat. Pura. Apl. (IMPA), Rio de Janeiro, 2005).

[26] C. Rosendal. Automatic continuity in homeomorphism groups of compact 2-manifolds. Israel J. Math. 166 (2008), 349-367.

[27] Christian Rosendal and S. Solecki. Automatic continuity of homomorphisms and fixed points on metric compacta. Israel J. Math. 162 (2007), 349-371.

[28] V. V. Ryzhikov. Representation of transformations preserving the Lebesgue measure, in the form of a product of periodic transformations. Math. Z. 38(6) (1985), 860-865, 957.

[29] A. Törnquist. Orbit equivalence and actions of $\mathbb{F}_{n}$. J. Symbolic Logic 71(1) (2006), 265-282.

[30] J. van Mill. Infinite-dimensional Topology (North-Holland Mathematical Library, 43). North-Holland, Amsterdam, 1989. 